\title{
Treatment of autoimmune diabetes in NOD mice by Toll-like receptor 2 tolerance in conjunction with dipeptidyl peptidase 4 inhibition
}

\author{
D.-H. Kim • J.-C. Lee • M.-K. Lee • K.-W. Kim • M.-S. Lee
}

Received: 10 May 2012 / Accepted: 9 August 2012 /Published online: 27 September 2012

(C) Springer-Verlag 2012

\begin{abstract}
Aims/hypothesis We have shown that chronic administration of the Toll-like receptor 2 (TLR2) agonist Pam $3 \mathrm{CSK}_{4}$ prevents diabetes in NOD mice by inducing TLR2 tolerance of dendritic cells (DCs). We have also reported that a novel dipeptidyl peptidase 4 (DPP4) inhibitor, DA-1229, could increase beta cell mass. Here we investigated whether a combination of DPP4 inhibition, with beneficial effects on beta cell mass, and TLR2 tolerisation, protecting beta cells from autoimmune destruction, could treat a model of established type 1 diabetes.

Methods Diabetic NOD mice were treated with $100 \mu \mathrm{g}$ Pam3CSK 4 , administered three times a week for 3 weeks, in combination with feeding with chow containing $0.3 \%$ DA-1229. Beta cell mass and proliferation were studied by immunohistochemistry. DC tolerance was assessed by studying diabetogenic $\mathrm{CD} 4^{+} \mathrm{T}$ cell priming after adoptive transfer and expression of costimulatory molecules on DCs by flow cytometry.
\end{abstract}

Electronic supplementary material The online version of this article (doi:10.1007/s00125-012-2723-x) contains peer-reviewed but unedited supplementary material, which is available to authorised users.

D.-H. Kim and J.-C. Lee contributed equally to this study.

D.-H. Kim • J.-C. Lee • M.-K. Lee · K.-W. Kim • M.-S. Lee $(\bowtie)$

Department of Medicine, Samsung Medical Center,

Sungkyunkwan University School of Medicine,

50 Irwon-dong, Kangnam-ku,

Seoul 135-710, Korea

e-mail: mslee0923@skku.edu

\section{M.-S. Lee}

Department of Medicine and Samsung Advanced Institute for

Health Sciences and Technology, Samsung Medical Center,

Sungkyunkwan University School of Medicine,

Seoul, Korea
Results We observed reversal of diabetes in NOD mice by $\mathrm{Pam}_{3} \mathrm{CSK}_{4}+\mathrm{DA}-1229$ but not by either $\mathrm{Pam}_{3} \mathrm{CSK}_{4}$ or DA1229 alone. Beta cell mass and the number of proliferating beta cells were significantly enhanced by $\mathrm{Pam}_{3} \mathrm{CSK}_{4}+\mathrm{DA}-$ 1229, but not by either Pam3CSK 4 or DA-1229 alone. Diabetogenic T cell priming by DCs and upregulation of costimulatory molecules after ex vivo stimulation were attenuated in mice treated with $\mathrm{Pam} 3 \mathrm{CSK}_{4}+\mathrm{DA}-1229$, indicating DC tolerance. The relative proportions of $\mathrm{CD} 4{ }^{+}$ $\mathrm{T}$ cells, $\mathrm{CD} 8^{+} \mathrm{T}$ cells, B cells, DCs, macrophages and regulatory $\mathrm{T}$ cells, and T-helper polarisation were unchanged by treatment with $\mathrm{Pam}_{3} \mathrm{CSK}_{4}+\mathrm{DA}-1229$.

Conclusions/interpretation These data demonstrate that a combination of TLR2 tolerisation and DPP4 inhibition can reverse early-onset diabetes in NOD mice.

Keywords Dipeptidyl peptidase $4 \cdot$ Immune tolerance . NOD $\cdot$ TLR2 $\cdot$ Treatment $\cdot$ Type 1 diabetes

$\begin{array}{ll}\text { Abbreviations } \\ \text { Ab } & \text { Antibody } \\ \text { CFSE } & \text { Carboxyfluorescein diacetate succinimidyl ester } \\ \text { DC } & \text { Dendritic cell } \\ \text { DPP4 } & \text { Dipeptidyl peptidase } 4 \\ \text { FOXP3 } & \text { Forkhead box P3 } \\ \text { GLP-1 } & \text { Glucagon-like peptide 1 } \\ \text { H\&E } & \text { Haematoxylin and eosin } \\ \text { IHC } & \text { Immunohistochemistry } \\ \text { iPS } & \text { Induced pleuripotential stem cell } \\ \text { MFI } & \text { Mean fluorescence intensity } \\ \text { MLN } & \text { Mesenteric lymph node } \\ \text { PLN } & \text { Pancreatic lymph node } \\ \text { STZ } & \text { Streptozotocin } \\ \text { TCR } & \text { T cell receptor } \\ \text { TLR2 } & \text { Toll-like receptor 2 } \\ \text { Treg } & \text { Regulatory T }\end{array}$




\section{Introduction}

It has been reported that activation of dendritic cells (DCs) by apoptotic beta cells during organogenesis of the pancreas and priming of autoreactive T cells by DCs in the pancreatic lymph nodes (PLNs) are the initial events in the NOD mouse model of type 1 diabetes $[1,2]$. We showed that apoptotic beta cells undergoing secondary necrosis, called 'late apoptotic' beta cells, can stimulate antigen-presenting cells through Toll-like receptor 2 (TLR2), contributing to the priming of diabetogenic T cells and the development of type 1 diabetes [3]. Recently, we reported that prolonged treatment with a TLR2 agonist inhibits the development of diabetes in NOD mice by inducing TLR2 tolerance [4]. Furthermore, prolonged treatment with a TLR2 agonist protected islet grafts from autoimmune destruction by sensitised T cells. These results suggest that induction of TLR2 tolerance may be able to treat established type 1 diabetes when combined with methods of enhancing beta cell mass, such as islet transplantation. However, the supply of islets for transplantation is limited, precluding widespread application of islet transplantation for the treatment of type 1 diabetes.

Strategies with potential beneficial effects on beta cell mass include glucagon-like peptide 1 (GLP-1) agonists [5, 6] or inhibitors of dipeptidyl peptidase 4 (DPP4) [7, 8], which degrades GLP-1 [9]. We have reported that a DPP4 inhibitor ameliorated diabetes induced by streptozotocin (STZ), and that this was attributable an increased beta cell mass [10]. However, DPP4 inhibition alone may not be able to reverse clinically overt type 1 diabetes as established autoimmunity against beta cells would destroy newly formed beta cells.

Because DPP4 inhibition could be one of the few currently available methods of increasing beta cell mass, and TLR2 tolerisation could inhibit the autoimmune response against beta cells, we studied the potential therapeutic value of combining TLR2 tolerisation and DPP4 inhibition for the treatment of established type 1 diabetes.

\section{Methods}

Cells and reagents $\mathrm{Pam}_{3} \mathrm{CSK}_{4}$ was from EMC Microcollections (Tübingen, Germany). DA-1229, a DPP4-selective inhibitor with an $\mathrm{IC}_{50}$ value of $0.9 \mathrm{nmol} / 1$ [10], was provided by Dong-A Pharmaceutical (Yong-In, Korea). All other chemicals were from Sigma (St Louis, MO, USA), unless indicated otherwise.

Mice NOD/Lt and BDC2.5/NOD T cell receptor (TCR)transgenic mice were from Jackson Laboratory (Bar Harbor, ME, USA). The incidence of diabetes in female and male NOD mice was about $70 \%$ and $30 \%$, respectively, at 24 weeks of age. BDC2.5 mice were typed by flow cytometry using monoclonal antibodies (Abs) against CD4 and V 34 (eBioscience, San Diego, CA, USA). Diabetes was monitored by weekly urine testing and confirmed by two consecutive measurements of non-fasting glucose above $13.9 \mathrm{mmol} / \mathrm{l}$ or a single measurement above $16.7 \mathrm{mmol} / \mathrm{l}$. All mice were maintained under specific pathogen-free conditions at the Samsung Medical Center Animal Facility. All animal experiments in this work were performed in accordance with the institutional guidelines.

ELISA Serum levels of insulin and GLP-1 were measured using ELISA kits (Shibayagi [Gumna, Japan] and ALPCO Diagnostics [Salem, NH, USA], respectively) according to the manufacturers' recommendations.

In vivo $T$ cell priming Diabetogenic $\mathrm{T}$ cell priming was assessed as described [2]. Briefly, $\mathrm{CD} 4^{+} \mathrm{T}$ cells were prepared from the pooled spleens of BDC2.5/NOD mice by the negative-selection method using a $\mathrm{CD} 4^{+} \mathrm{T}$ cell isolation kit (Miltenyl Biotech, Bergisch Gladbach, Germany) (purity $>95 \%$ ). Carboxyfluorescein diacetate succinimidyl ester (CFSE) (Molecular Probes, Eugene, OR, USA)-labelled BDC2.5 cells $\left(2 \times 10^{6}\right)$ were transferred by tail-vein injection into NOD mice treated with Pam3CSK 4 and/or DA-1229 for 3 weeks. The PLNs, mesenteric lymph nodes (MLNs) and spleens were harvested $66 \mathrm{~h}$ after transfer, and single-cell suspensions were analysed for CFSE dilution by flow cytometry gated on $\mathrm{CD}^{+}$and $\mathrm{V} \beta 4^{+}$cells.

DC tolerance To study DC tolerance, mononuclear cells were isolated from the spleens and PLNs of NOD mice treated with Pam $3 \mathrm{CSK}_{4}+\mathrm{DA}-1229$ for 3 weeks, and incubated with $\mathrm{Pam}_{3} \mathrm{CSK}_{4}$ ex vivo for 1 day. The expression of CD80, CD83 and CD86 costimulatory molecules on DCs was determined by flow cytometry gated on $\mathrm{CD} 11 \mathrm{c}^{+}$cells after incubation with specific Abs (eBioscience). To study DC activation by chronic $\mathrm{Pam} \mathrm{CSK}_{4}$ administration in vivo, splenocytes were freshly isolated from mice treated with Pam $3 \mathrm{CSK}_{4}+\mathrm{DA}-1229$ for 3 weeks, and the expression of CD80, CD83 and CD86 on DCs was determined as above without ex vivo $\mathrm{Pam}_{3} \mathrm{CSK}_{4}$ treatment.

Regulatory $T$ cells The percentage of regulatory $\mathrm{T}$ (Treg) cells was analysed by triple-coloured flow cytometry after staining with anti-CD4, -CD25 and -mouse/rat forkhead box P3 (FOXP3) (FJK-16s) Abs, as described [3, 4]. Pancreatic islets were prepared as described [11] and incubated in $0.05 \%$ (wt/vol.) trypsin- $0.02 \%$ (wt/vol.) EDTA solution at $37^{\circ} \mathrm{C}$ for $5 \mathrm{~min}$ for dissociation into single islet cells and mononuclear cells.

Th1/Th2/Th17 subsets Mononuclear cells from the spleens and PLNs $\left(1 \times 10^{6}\right)$ were stimulated with phorbol myristate 
acetate/ionomycin (BD Biosciences, San Jose, CA, USA)/ brefeldin A (eBioscience) for $5 \mathrm{~h}$. After surface staining using anti-CD4 Ab, cells were fixed and permeabilised with Cytofix/Cytoperm (BD Biosciences). Subsequently, cells were stained intracellularly with anti-IFN- $\gamma \mathrm{Ab}$ (eBioscience) together with anti-IL-4 (eBioscience) or -IL-17 Ab (eBioscience). Samples were acquired on a FACSCalibur equipped with CellQuest software (BD Biosciences), and data were analysed using a FlowJo software (Tree Star, Ashland, OR, USA).

Histological analysis After fixation and embedding, the pancreases were sectioned to a thickness of $3 \mu \mathrm{m}$ for haematoxylin and eosin (H\&E) staining. For insulitis scoring, more than 30 pancreatic islets from more than three parallel sections were examined per mouse, and the degree of insulitis was graded as described [11]. Islet size was determined using a digital camera, and analysis of each islet was performed using an image analyser.

Analysis of beta and alpha cells Insulin immunohistochemistry (IHC) was done using guinea pig anti-porcine insulin $\mathrm{Ab}$ (DAKO, Carpinteria, CA, USA) as described [12]. Relative beta cell mass was estimated by point counting after insulin IHC [13]. An average of 7,000 points/mouse was counted. BrdU incorporation into beta cells was evaluated $16 \mathrm{~h}$ after i.p. injection of $100 \mathrm{mg} / \mathrm{kg}$ BrdU by double IHC using anti-insulin and -BrdU Abs (Serotec, Raleigh, NC, USA) [12]. The number of insulin ${ }^{+} \mathrm{BrdU}^{+}$beta cells was normalised to the total islet area measured using the Axion Vision 4 software (Zeiss, Göttingen, Germany). The number of small beta cell units (beta cell cluster comprising three insulin $^{+}$cells at a maximum [14]) was normalised to the total pancreas area. Relative alpha cell mass was measured by point counting after glucagon IHC using a specific $\mathrm{Ab}$ (Cell Signaling, Danvers, MA, USA). Beta/alpha cell mass ratio was calculated after IHC of consecutive sections using respective specific Abs.

Statistical analysis All values are expressed as the means \pm SE. A two-tailed Student's $t$ test was employed to compare values between two groups. One-way ANOVA was employed to compare values among multiple groups. Once the ANOVA test showed significant differences, the Duncan post hoc test was employed to compare two specific groups. When the Duncan test showed no significant differences $(p>0.05)$, the two groups were labelled with the same letter over their respective bars. Distribution of islet size was compared between groups using the $\chi^{2}$ test. The proportion of islets of individual size was compared between groups using the binominal test. $p$ values $<0.05$ were considered significant.

\section{Results}

Reversal of the model of established type 1 diabetes by TLR2 agonist + DPP4 inhibitor We first studied whether TLR2 tolerance and/or DPP4 inhibition could reverse the model of established type 1 diabetes in NOD mice. Administration of $100 \mu \mathrm{g} \mathrm{Pam} 3 \mathrm{CSK}_{4}$ i.p. three times a week for 3 weeks, which induces prolonged TLR2 tolerance [4], to nine female diabetic NOD mice with non-fasting blood glucose levels of 13.9 19.4 mmol/1 failed to restore normoglycaemia in eight mice (normoglycaemia, 11\%) (Fig. 1a). This probably occurred because inhibition of autoimmunity alone cannot restore critically reduced beta cell mass. Feeding female diabetic NOD mice with non-fasting blood glucose levels of $13.9 \sim 19.4 \mathrm{mmol} / 1$ with $0.3 \%$ DA-1229containing chow also failed to reverse established diabetes in eight of eight mice (normoglycaemia, $0 \%$ ) (Fig. 1a). This probably occurred because DA-1229 alone cannot inhibit autoimmunity despite its potential beneficial effect on beta cell mass [10]. Moreover, feeding 0.3\% DA-1229containing chow alone did not inhibit the development of diabetes in NOD mice (electronic supplementary material [ESM] Fig. 1), supporting the finding that DA-1229 alone cannot inhibit autoimmunity. In contrast, when female diabetic NOD mice with non-fasting blood glucose levels of 13.9 19.4 mmol/l were treated with $100 \mu \mathrm{g} \mathrm{Pam}_{3} \mathrm{CSK}_{4}$ administration three times a week for 3 weeks in combination with feeding with $0.3 \%$ DA-1229-containing chow, normoglycaemia was achieved in ten of 12 mice $(83 \%)$ (Fig. 1b). The mean non-fasting blood glucose level was significantly decreased by treatment with $\mathrm{Pam}_{3} \mathrm{CSK}_{4}+\mathrm{DA}$ 1229 for 3 weeks, while the non-fasting blood glucose level was increased or unchanged in diabetic NOD mice untreated for 3 weeks and those treated with either Pam3CSK 4 or DA1229 alone for 3 weeks (Fig. 1c). The non-fasting blood glucose level was further decreased by treatment with Pam $3 \mathrm{CSK}_{4}+\mathrm{DA}-1229$ for 15 weeks, and was significantly lower compared with the same treatment for 3 weeks (Fig. 1c). Blood glucose levels at 15 weeks could not be determined in untreated diabetic NOD mice or those treated with either Pam3CSK 4 or DA-1229 alone because those mice were humanely killed before 15 weeks because of the deterioration in their health. In mice showing amelioration of blood glucose profile after treatment with Pam3CSK ${ }_{4}+$ DA-1229, insulinogenic index $\left(\Delta\right.$ insulin $_{15}$ min $/$ $\Delta$ glucose $_{15} \mathrm{~min}$ ), representing beta cell function [15], was significantly enhanced compared with untreated diabetic NOD mice and those treated with either Pam $3 \mathrm{CSK}_{4}$ or DA-1229 alone for 3 weeks, and was not different from that in prediabetic NOD mice (Fig. 1d). In contrast, serum levels of GLP-1 were elevated in diabetic NOD mice treated with Pam $3 \mathrm{CSK}_{4}+\mathrm{DA}-1229$ and those treated with DA-1229 alone for 3 weeks compared with untreated diabetic NOD 

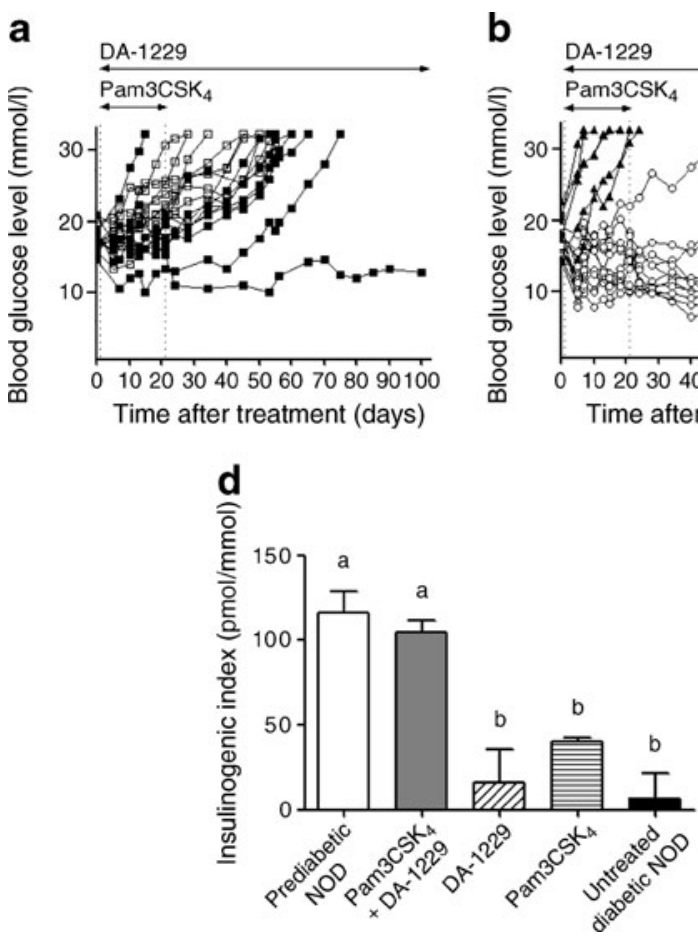

Fig. 1 Reversal of established diabetes of NOD mice by $\mathrm{Pam}_{3} \mathrm{CSK}_{4}$ in combination with DA-1229, a DPP4 inhibitor. (a,b) In diabetic NOD mice with non-fasting blood glucose levels of 13.9 16.7 mmol/1, $100 \mu \mathrm{g} \mathrm{Pam} 3 \mathrm{CSK}_{4}$ was injected i.p. three times a week for 3 weeks and animals were fed chow containing 0.3\% DA-1229 throughout the experimental period (b, white circle, $n=12)$. As controls, diabetic NOD mice were untreated (b, black triangle, $n=6)$ or treated with either i.p. injection of $100 \mu \mathrm{g} \mathrm{Pam}_{3} \mathrm{CSK}_{4}$ three times a week for 3 weeks (a, black square, $n=9$ ) or feeding with chow containing $0.3 \%$ DA1229 alone (a, white square, $n=8$ ). Blood glucose level was monitored weekly. (c) Non-fasting blood glucose levels in diabetic NOD mice treated with $\mathrm{Pam}_{3} \mathrm{CSK}_{4}$ and/or DA-1229 were measured before and after 3 or 15 weeks of treatment (grey bar, $\mathrm{Pam}_{3} \mathrm{CSK}_{4}+\mathrm{DA}-1229, n=7$; horizontally hatched bar, $\mathrm{Pam} 3 \mathrm{CSK}_{4}, n=7$; diagonally hatched bar, DA-1229, $n=6$; black bar, untreated diabetic NOD, $n=5$ ). (d) After

mice and those treated with $\mathrm{Pam}_{3} \mathrm{CSK}_{4}$ alone for 3 weeks (Fig. 1e). These results suggest that feeding 0.3\% DA-1229containing chow effectively inhibited DPP4 in vivo, regardless of Pam $3 \mathrm{CSK}_{4}$ administration or blood glucose profile, and supports the suggestion that GLP-1 elevation alone cannot restore normoglycaemia in diabetic NOD mice.

In contrast to these results, we failed to reverse the model of established diabetes in NOD mice with Pam3CSK 4 +DA-1229 when non-fasting glucose levels were above $19.4 \mathrm{mmol} / \mathrm{l}$ (data not shown). This suggests that our method can be applied to established type 1 diabetes when substantial numbers of beta cells remain but not when extensive beta cell damage has already reached a certain threshold.

Beta cells in NOD mice treated with TLR2 agonist + DPP4 inhibitor We next studied the morphological changes of pancreatic islets. While diabetic NOD mice treated with Pam $3 \mathrm{CSK}_{4}+\mathrm{DA}-1229$ for 3 weeks had insulitis, the insulitis
C

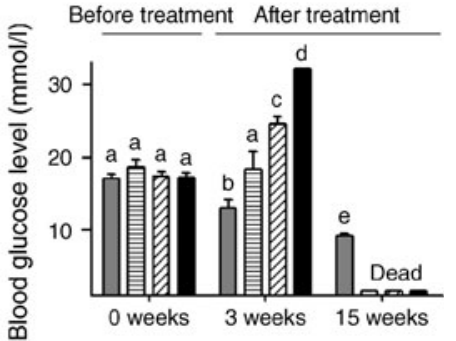

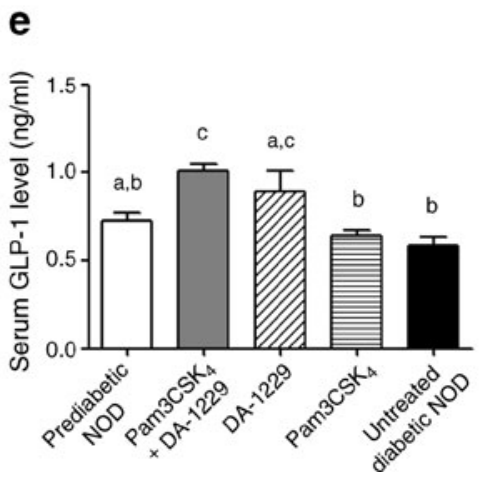

3 weeks of treatment with $\mathrm{Pam}_{3} \mathrm{CSK}_{4}$ and/or DA-1229, serum was obtained before and $15 \mathrm{~min}$ after $1 \mathrm{~g} / \mathrm{kg}$ glucose injection i.p. to overnight fasted mice for measurement of insulin level by ELISA and calculation of insulinogenic index. Serum insulin level was also measured in 15-week-old prediabetic NOD mice and diabetic NOD mice untreated for 3 weeks $(n=4$ each). (e) After 3 weeks of treatment with Pam3CSK 4 and/or DA-1229, the fasting GLP-1 level was determined by ELISA. Serum GLP-1 level was also measured in 15 -weekold prediabetic NOD mice and diabetic NOD mice untreated for 3 weeks ( $n=4$ each). The same letter above two bars indicates that the two values are not different from each other by the Duncan post hoc test $(p>0.05)$. Conversely, in the absence of the same letter above two bars, the two data points are significantly different by the Duncan test $(p<0.05)$

score was significantly lower compared with that of untreated diabetic NOD mice and those treated with either $\mathrm{Pam}_{3} \mathrm{CSK}_{4}$ or DA-1229 alone for 3 weeks, and was not different from that in 15-week-old prediabetic NOD mice (Fig. 2a, b). The attenuation of the insulitis score by Pam $3 \mathrm{CSK}_{4}+\mathrm{DA}-1229$ is probably caused by protection of the remaining beta cells and newly formed beta cells from autoimmune destruction (see below), which would reduce the severity of insulitis. When we examined the distribution of islet size, the percentage of islets with a large size $\left(>6,000 \mu \mathrm{m}^{2}\right)$, which account for a major portion of beta cell function, was significantly higher in diabetic NOD mice treated with $\mathrm{Pam}_{3} \mathrm{CSK}_{4}+\mathrm{DA}-1229$ compared with untreated diabetic NOD mice and was similar to that in prediabetic NOD mice (Fig. 2c).

We next measured beta cell mass as an indicator of beta cell function. Insulin IHC followed by point counting showed that beta cell mass was significantly higher in 

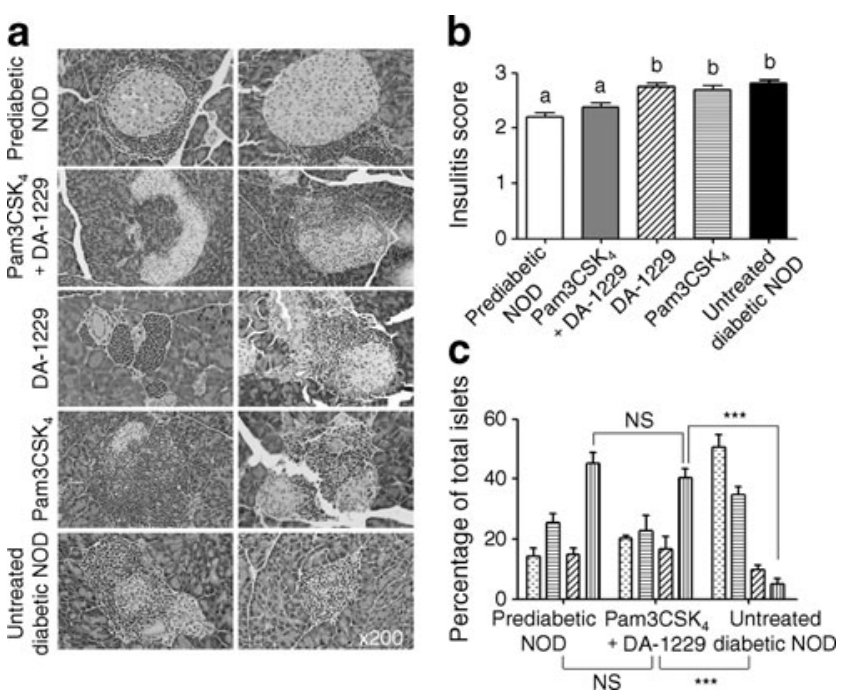

Fig. 2 Islet histology of diabetic NOD mice treated with Pam $3 \mathrm{CSK}_{4}$ in combination with DA-1229. (a) Pancreas tissue was harvested from diabetic NOD mice after treatment with Pam $3 \mathrm{CSK}_{4}$ and/or DA-1229 for 3 weeks. Islet sections were also prepared from 15 -week-old female prediabetic NOD mice and diabetic NOD mice untreated for 3 weeks. Representative H\&E sections are shown. (b) Insulitis scoring was done as described in the Methods ( $n=3$ each). (c) Islet size in 15 -week-old prediabetic NOD mice, diabetic NOD mice treated with Pam $3 \mathrm{CSK}_{4}+\mathrm{DA}-1229$ for 3 weeks and diabetic NOD mice untreated for 3 weeks was determined as described in the Methods ( $n=3$ each) (dotted bar, 1-1,000 $\mathrm{mm}^{2}$; horizontally hatched bar, 1,001-3,000 $\mu \mathrm{m}^{2}$; diagonally hatched bar, 3,001-6,000 $\mathrm{mm}^{2}$; vertically hatched bar, $>6,001 \mu^{2}$ ). The distribution of islet size in diabetic NOD mice treated with Pam $3 \mathrm{CSK}_{4}+\mathrm{DA}-1229$ for 3 weeks was significantly different from that in untreated diabetic NOD mice, and was similar to that in prediabetic NOD mice, as tested by $\chi^{2}$ test. The absence of the same letter above two bars indicates that the two data points are significantly different by the Duncan test $(p<0.05) .{ }^{* * *} p<0.001$

NOD mice treated with Pam3CSK 4 +DA-1229 for 3 weeks compared with untreated diabetic NOD mice and those treated with DA-1229 alone for 3 weeks, and was not significantly different from that in 15 -week-old prediabetic NOD mice (Fig. 3a, b), probably because newly formed beta cells were protected from autoimmune destruction. Beta cell mass in NOD mice treated with $\mathrm{Pam}_{3} \mathrm{CSK}_{4}+\mathrm{DA}-1229$ for 3 weeks appeared higher compared with diabetic NOD mice treated with $\mathrm{Pam}_{3} \mathrm{CSK}_{4}$ alone, but the difference was insignificant, probably because of a large variance (Fig. 3b).

To study the mechanism of the increased beta cell mass, we assessed beta cell proliferation that could be upregulated by DPP4 inhibitors $[6,10]$ by quantifying the number of beta cells incorporating $\mathrm{BrdU}$. The $\mathrm{BrdU}^{+}$beta cell number was significantly higher in NOD mice treated with Pam $3 \mathrm{CSK}_{4}+\mathrm{DA}-1229$ for 3 weeks compared with untreated diabetic NOD mice and those treated with either Pam3CSK 4 or DA-1229 alone for 3 weeks (Fig. 3d, e). The number of proliferating beta cells in diabetic NOD mice treated with either Pam3CSK 4 or DA-1229 alone for 3 weeks was not a

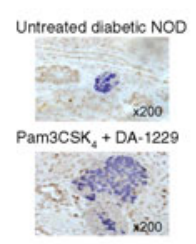

d

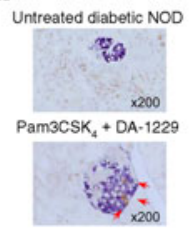

g

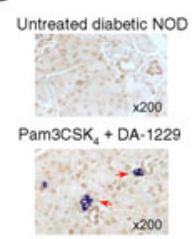

b

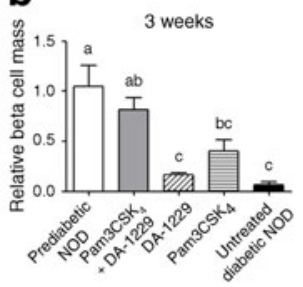

e

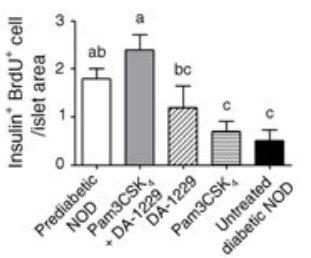

h

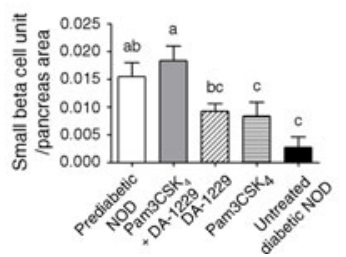

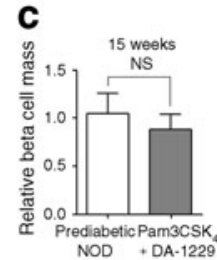

f

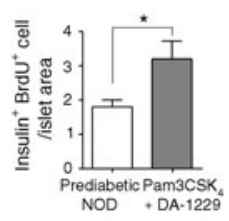

Fig. 3 Relative beta cell mass and beta cell proliferation in diabetic NOD mice treated with $\mathrm{Pam}_{3} \mathrm{CSK}_{4}+\mathrm{DA}-1229$. (a) Representative images of insulin IHC of pancreas tissue sections harvested from groups of mice as in Fig. 2a. (b,c) Relative beta cell mass was assessed by the point-counting method as described in the Methods after: (b) treatment for 3 weeks; and (c) treatment for 15 weeks. (d-f) At $16 \mathrm{~h}$ after i.p. injection of $100 \mathrm{mg} / \mathrm{kg} \mathrm{BrdU}$, pancreatic sections were prepared as in (a) for double IHC using anti-insulin and -BrdU Abs to measure beta cell proliferation. (d) Representative images of IHC; arrows indicate representative BrdU-incorporating beta cells. (e,f) Number of beta cells incorporating BrdU per islet area after: (e) treatment for 3 weeks; and (f) treatment for 15 weeks. (g-i) Insulin $\mathrm{IHC}$ was done as in (a) to determine the number of small beta cell units comprising three insulin ${ }^{+}$cells at a maximum. (g) Representative images of IHC; arrows indicate representative small beta cell units. (h, i) Number of small beta cell units per pancreas area after: (h) treatment for 3 weeks; and (i) treatment for 15 weeks. In (b), (e) and (h), the absence of the same letter above two bars indicates that the two data points are significantly different by the Duncan test $(p<0.05)$. $n=3 \sim 4$ each; ${ }^{*} p<0.05$

different from that in untreated diabetic NOD mice (Fig. 3e), probably because both increased beta cell proliferation and protection from autoimmunity are necessary to increase the number of proliferating beta cells. We also studied small beta cell units, comprising three insulin ${ }^{+}$cells at a maximum, that could represent beta cell neogenesis [14]. The number of small beta cell units was significantly elevated in NOD mice treated with $\mathrm{Pam}_{3} \mathrm{CSK}_{4}+\mathrm{DA}-1229$ for 3 weeks compared with untreated diabetic NOD mice and those treated with either $\mathrm{Pam}_{3} \mathrm{CSK}_{4}$ or DA-1229 alone for 3 weeks (Fig. 3g, h), again because both increased numbers 
of small scattered beta cells and their protection from autoimmunity are necessary for an increased number of small beta cell units. Thus, the number of small beta cell units in diabetic NOD mice treated with either Pam $3 \mathrm{CSK}_{4}$ or DA1229 alone for 3 weeks was not different from that in untreated diabetic NOD mice (Fig. 3h).

We also studied beta cell changes after treatment for 15 weeks to study the long-term effect. Beta cell mass in diabetic NOD mice treated with $\mathrm{Pam}_{3} \mathrm{CSK}_{4}+\mathrm{DA}-1229$ for 15 weeks was not different from that in prediabetic 15 week-old NOD mice (Fig. 3c). The numbers of proliferating beta cells and small beta cell units were significantly elevated in diabetic NOD mice treated with Pam3CSK 4 +DA-1229 for 15 weeks compared with prediabetic NOD mice (Fig. 3f, i), probably because of the elevated GLP-1 levels in treated NOD mice compared with prediabetic NOD mice without DA-1229 administration (Fig. 1d). The numbers of proliferating beta cells and small beta cell units appeared elevated in diabetic NOD mice treated with Pam $3 \mathrm{CSK}_{4}+\mathrm{DA}-1229$ for 3 weeks compared with prediabetic NOD mice; however, the difference was statistically insignificant (Fig. 3e, h), probably because of the short duration of treatment.

We also studied possible changes in alpha cells that could be affected by DPP4 inhibitors [7] and could influence glucose profile of diabetic mice. Contrary to the widespread conception that beta cells are selectively destroyed in type 1 diabetes, alpha cell mass was significantly reduced in untreated diabetic NOD mice compared with prediabetic NOD mice (ESM Fig. 2a, b). In NOD mice treated with Pam $3 \mathrm{CSK}_{4}+\mathrm{DA}-1229$ for 3 weeks, alpha cell mass was significantly increased compared with untreated diabetic NOD mice, while only insignificant changes were noted in mice treated with either Pam3CSK 4 or DA-1229 alone for 3 weeks (ESM Fig. 2a, b). Beta/alpha cell mass ratio was significantly lower in untreated diabetic NOD mice compared with prediabetic NOD mice, suggesting that beta cells are predominant targets of autoimmunity in NOD mice despite some alpha cell changes occurring in NOD mice (ESM Fig. 2c). The reduced beta/alpha cell mass ratio was normalised by combined treatment with $\mathrm{Pam}_{3} \mathrm{CSK}_{4}+\mathrm{DA}-$ 1229 for 3 weeks, but not by treatment with either Pam3CSK 4 or DA-1229 alone (ESM Fig. 2c). These results show that DPP4 inhibition alone did not significantly increase alpha cell mass in diabetic NOD mice but did increase it when combined with TLR2 tolerisation, and suggest that the effect of DPP4 inhibition on alpha cell mass is secondary to beta cell changes, as alpha cells are not major targets of autoimmunity and are not likely to be affected by the changes of immune function associated with TLR2 tolerisation.

DC tolerance in NOD mice treated with TLR2 agonist +DPP4 inhibitor We next investigated whether the increased beta cell mass in diabetic NOD mice treated with Pam $3 \mathrm{CSK}_{4}+\mathrm{DA}-1229$ is accompanied by TLR2 tolerance of DCs. When we measured the proliferation of infused BDC2.5 $\mathrm{CD}^{+}{ }^{+} \mathrm{T}$ cells harbouring transgene-encoded $\mathrm{Tcr}$ $\alpha /$ Tcr- $\beta$ (also known as Tcra/Tcrb) genes derived from a diabetogenic $\mathrm{CD} 4^{+} \mathrm{T}$ cell clone reflecting autoreactive $\mathrm{T}$ cell priming by DCs [2], successive dilutions of the CFSE label in BDC2.5 $\mathrm{CD}^{+}{ }^{+} \mathrm{T}$ cells were observed in the PLNs (Fig. 4a, b), but not in the MLNs or spleens of prediabetic NOD mice (ESM Fig. 3), showing proliferation of diabetogenic $\mathrm{CD}^{+} \mathrm{T}$ cells specifically in the PLNs as reported [2]. A similar pattern of BDC2.5 $\mathrm{CD}^{+} \mathrm{T}$ cell proliferation was observed in the PLNs, MLNs and spleens of diabetic NOD mice treated with DA-1229 alone (Fig. 4a, b; ESM Fig. 3), suggesting that DPP4 inhibition does not affect diabetogenic $\mathrm{T}$ cell priming by DCs. In NOD mice treated with $\mathrm{Pam}_{3} \mathrm{CSK}_{4}$ alone or Pam3CSK $4+\mathrm{DA}-1229$ combination, the proliferation of $\mathrm{BDC} 2.5 \mathrm{CD}^{+} \mathrm{T}$ cells in the PLNs was significantly reduced compared with that in prediabetic NOD mice (Fig. 4a, b). These results suggest that TLR2 tolerance of DCs was induced in NOD mice treated with Pam3CSK 4 +DA-1229, independent of DA-1229 [4], and protected the remaining beta cells and new beta cells from autoimmune destruction, leading to the restoration of normoglycaemia in diabetic NOD mice.

We further studied the mechanism of DC tolerance in mice treated with Pam3CSK 4 +DA-1229. When lymphocytes from the spleens or PLNs were incubated with $100 \mathrm{ng} / \mathrm{ml}$ $\mathrm{Pam}_{3} \mathrm{CSK}_{4}$ ex vivo for 1 day, the increases in the expression level of costimulatory molecules such as CD80, CD83 or

Fig. 4 DC tolerance in NOD mice treated with Pam3CSK 4 +DA-1229. $(\mathbf{a}, \mathbf{b}) \mathrm{CD}^{+} \mathrm{T}$ cells prepared from BDC2.5/NOD mice by the negativeselection method were labelled with CFSE and transferred by tail-vein injection into NOD mice treated with $\mathrm{Pam}_{3} \mathrm{CSK}_{4}$ and/or DA-1229 for 3 weeks or prediabetic NOD mice. $\mathrm{CD} 4{ }^{+} \mathrm{T}$ cell proliferation in the PLNs was assessed by analysing CFSE dilution by flow cytometry gated on $\mathrm{CD}^{+}$and $\mathrm{V} \beta 4^{+}$cells. (b) Results pooled from four independent experiments are presented. (a) Representative histograms. (c, d) Mononuclear cells were prepared from the spleens (c) and PLNs (d) of NOD mice treated with $\mathrm{Pam} 3 \mathrm{CSK}_{4}+\mathrm{DA}-1229$ for 3 weeks in vivo or prediabetic NOD mice. Cells were further incubated with $\mathrm{Pam}_{3} \mathrm{CSK}_{4}$ (grey graph) or PBS (white graph) ex vivo for 1 day, and the expression of costimulatory molecules on DCs was determined by flow cytometry gated on CD11 $\mathrm{c}^{+}$ cells. Changes in MFI on ex vivo incubation with $\mathrm{Pam}_{3} \mathrm{CSK}_{4}$ are shown in the bar charts (white bar, prediabetic NOD; grey bar, $\mathrm{Pam}_{3} \mathrm{CSK}_{4}+\mathrm{DA}-$ 1229); $n=3$ each. Significantly lower fold changes in MFI in mice treated with Pam $3 \mathrm{CSK}_{4}+\mathrm{DA}-1229$ for 3 weeks suggest that low inducibility of costimulatory molecules in DCs is an important feature of DC tolerance in those mice. (e,f) Mononuclear cells were isolated from the spleens (e) and PLNs (f) of NOD mice treated with $\mathrm{Pam}_{3} \mathrm{CSK}_{4}+\mathrm{DA}-1229$ for 3 weeks (grey line) or prediabetic NOD mice (black line), and the expression of costimulatory molecules on DCs was determined as in (c) without ex vivo Pam3CSK 4 treatment. MFI values are shown in the bar charts (white bar, prediabetic NOD; grey bar, $\mathrm{Pam}_{3} \mathrm{CSK}_{4}+\mathrm{DA}-1229$ ), $n=3$ each. The absence of the same letter above two bars indicates that the two data are significantly different by the Duncan test $(p<0.05) .{ }^{*} p<0.05$, ${ }^{* *} p<0.01$ 
a
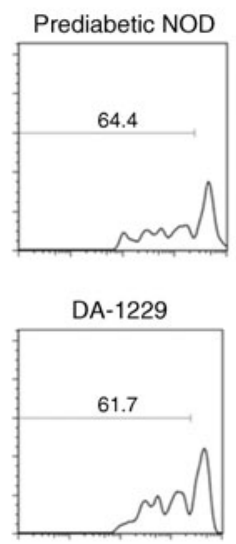

$\mathrm{Pam} 3 \mathrm{CSK}_{4}+\mathrm{DA}-1229$

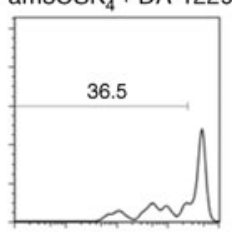

$\mathrm{Pam}_{3 \mathrm{CSK}} \mathrm{S}_{4}$

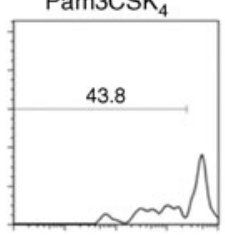

Gated on $\mathrm{CD}^{+}{ }^{+} \mathrm{V} \beta 4^{+}$cells

C CD80 CD83
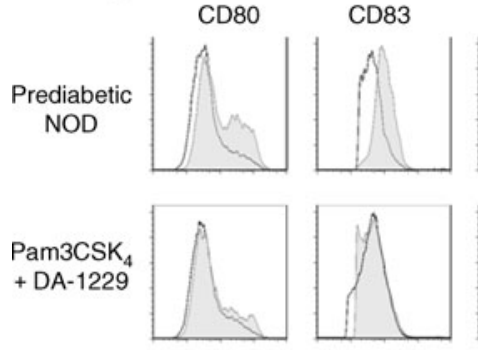

CD86
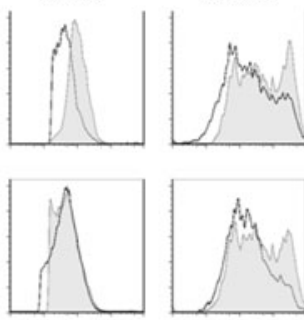

Gated on CD $11 c^{+}$cells

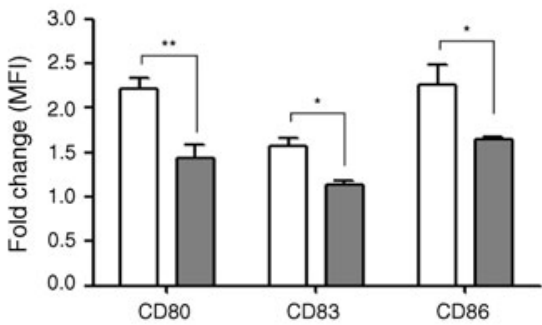

e
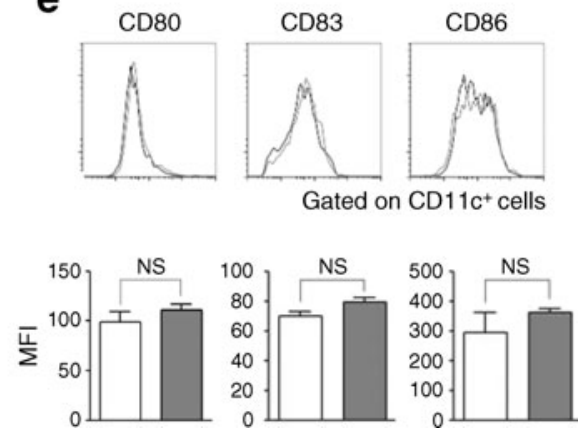

b

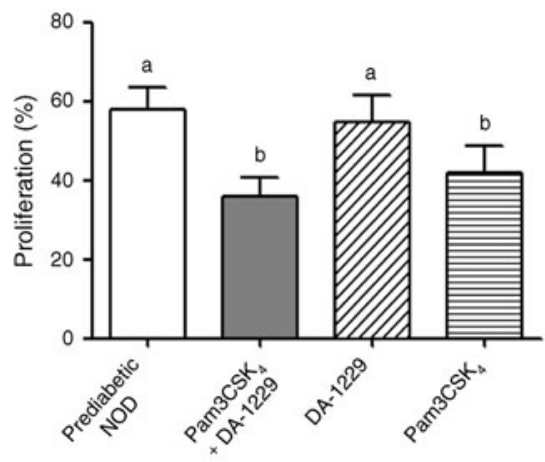

d

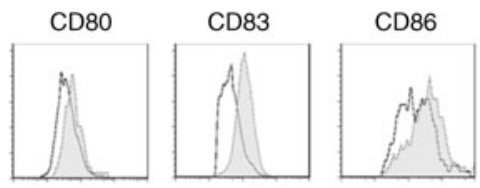

Prediabetic

NOD
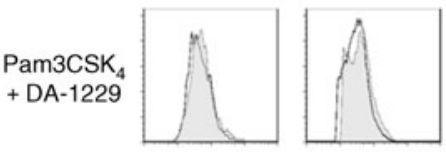

Gated on CD11c+ cells

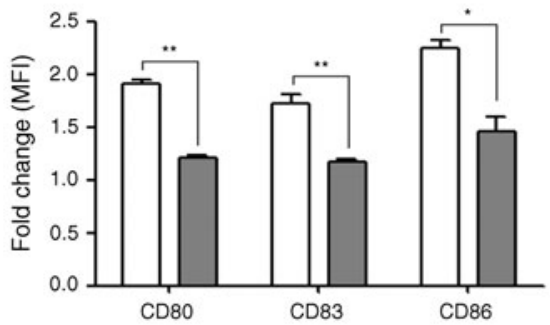

f
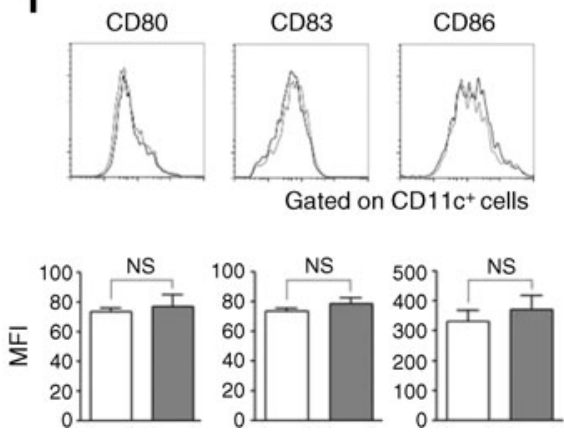

CD86 with ex vivo $\mathrm{Pam}_{3} \mathrm{CSK}_{4}$ treatment were less pronounced in mice treated with $\mathrm{Pam}_{3} \mathrm{CSK}_{4}+\mathrm{DA}-1229$ in vivo for 3 weeks compared with prediabetic NOD mice, as reflected by significantly lower fold increases in the mean fluorescence intensities (MFIs) in the treated mice compared with the prediabetic NOD mice (Fig. 4c, d). However, the expression of CD80, CD83 or CD86 in DCs from the spleens or PLNs of NOD mice treated with $\mathrm{Pam}_{3} \mathrm{CSK}_{4}+\mathrm{DA}-1229$ in vivo but without further Pam3CSK 4 treatment ex vivo was not different from that in DCs of prediabetic NOD mice (Fig. 4e, f), suggesting that prolonged treatment with $\mathrm{Pam} 3 \mathrm{CSK}_{4}+\mathrm{DA}-1229$ itself does not induce DC activation, similar to the findings in NOD mice treated with $\mathrm{Pam}_{3} \mathrm{CSK}_{4}$ alone [4].

No alteration of Treg cells and Th1/Th2/Th17 polarisation by TLR2 agonist + DPP4 inhibitor We next studied the 
possible changes in the immunological profile of NOD mice treated with $\mathrm{Pam} 3 \mathrm{CSK}_{4}+\mathrm{DA}-1229$. The relative proportions of $\mathrm{CD}^{+}{ }^{+}$and $\mathrm{CD} 8^{+}$T cells, $\mathrm{B} 220^{+} \mathrm{B}$ cells, $\mathrm{CD} 11 \mathrm{c}^{+} \mathrm{DCs}$ and $\mathrm{F} 4 / 80^{+} \mathrm{CD} 11 \mathrm{~b}^{+}$macrophages in the spleen, MLNs and PLNs were not significantly different between NOD mice treated with Pam $3 \mathrm{CSK}_{4}+\mathrm{DA}-1229$ for 3 weeks and 15-week-old prediabetic NOD mice (ESM Fig. 4). The proportion of $\mathrm{CD}^{+} \mathrm{FOXP}^{+}$Treg cells in the spleen, PLNs and pancreatic islets and the MFI of CD25 on $\mathrm{CD} 4^{+} \mathrm{FOXP} 3^{+}$Treg cells, reflecting their peripheral suppressive function [16], were also not significantly different between NOD mice treated with $\mathrm{Pam} 3 \mathrm{CSK}_{4}+\mathrm{DA}-1229$ and prediabetic NOD mice (Fig. 5a, b), which is similar to the findings in NOD mice treated with $\mathrm{Pam}_{3} \mathrm{CSK}_{4}$ alone [4]. The proportions of $\mathrm{CD} 4^{+} \mathrm{IFN}-\gamma^{+}$Th1 cells, CD $4^{+} \mathrm{IL}-4^{+}$Th2 cells or CD $4^{+} \mathrm{IL}-$ $17^{+}$Th17 cells in the spleens and PLNs of NOD mice treated with Pam $3 \mathrm{CSK}_{4}+\mathrm{DA}-1229$ were also not different from those in prediabetic NOD mice (Fig. 5c, d), again similar to the findings in NOD mice treated with Pam $3 \mathrm{CSK}_{4}$ alone [4]. These results suggest that reversal of diabetes by Pam $3 \mathrm{CSK}_{4}+\mathrm{DA}-1229$ is unrelated to the alteration of Treg cells or Th1/Th2/Th17 skewing.

\section{Discussion}

For the treatment of established type 1 diabetes, first, autoimmunity against beta cells should be inhibited, and, second, critically reduced beta cell mass should be replenished. Both goals are not easy to achieve using the techniques currently available for patients. Numerous immunological methods have been employed to inhibit autoimmune responses to beta cells in human or animal models of type 1 diabetes [17-27]. However, immunological methods alone were largely unsuccessful in the treatment of clinically overt type 1 diabetes, probably because of the already significantly compromised beta cell mass. Thus, strategies to replenish the reduced beta cell mass will be required for the treatment of type 1 diabetes patients.

Current methods of increasing beta cell mass are generally not satisfactory. Pancreas or islet transplantation could be the most effective method to increase beta cell mass if further auto- and allo-immune destruction can be suppressed. However, the supply of pancreases and islets from humans is limited. The use of tissue from non-human sources for xenotransplantation presents several obstacles, such as endogenous virus or hyperacute rejection. Recently, derivation of beta cells from induced pleuripotential stem cells (iPSs) has emerged as a promising technology to obviate the ethical problems associated with embryonic stem cells and alloimmune responses [28]. However, safety issues such as teratoma formation and low reprogramming efficiency preclude clinical application of iPS-derived beta cell technology. Other methods increasing

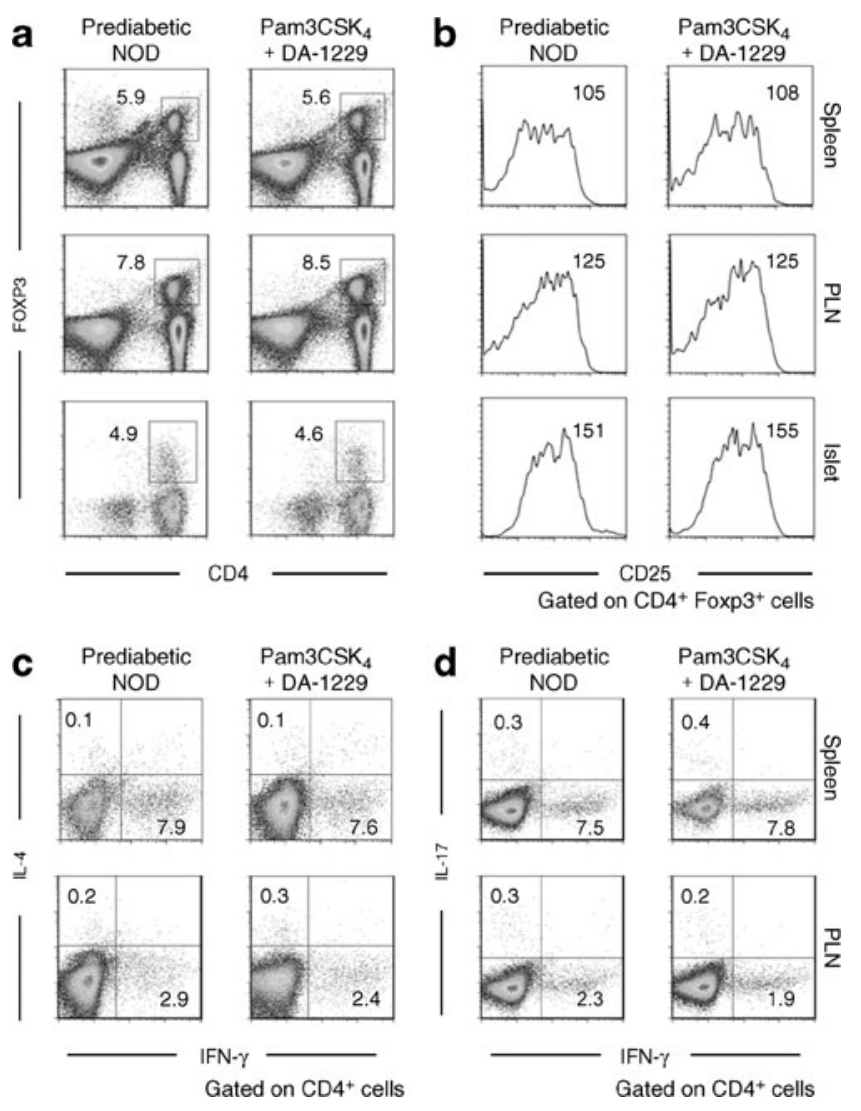

Fig. 5 Unaltered Treg cells and Th1/Th2/Th17 polarisation in NOD mice treated with $\mathrm{Pam}_{3} \mathrm{CSK}_{4}+\mathrm{DA}-1229$. (a,b) Mononuclear cells from the spleens and PLNs, and islets of NOD mice that were treated with $\mathrm{Pam}_{3 \mathrm{CSK}}+\mathrm{DA}-1229$ for 3 weeks or prediabetic NOD mice were stained using anti-CD4,-CD25 and -FOXP3 monoclonal Abs for triple-coloured flow cytometry as described in the Methods. The numbers in (a) represent the percentage of $\mathrm{CD} 4^{+} \mathrm{FOXP} 3^{+}$Treg cells. Those in (b) denote the MFIs of CD25 on $\mathrm{CD}^{+}{ }^{+} \mathrm{FOXP} 3^{+}$cells. $(\mathbf{c}, \mathbf{d})$ Th1/Th2/Th17 skewing of mononuclear cells from the spleen or PLNs of NOD mice treated with Pam3CSK 4 +DA-1229 or prediabetic NOD mice was examined as described in the Methods. The results are representative of three or four independent experiments showing similar tendencies

compromised beta cell mass comprise diverse pharmacological agents or hormones, including GLP-1 and DPP4 inhibitors [29-36]. In mice treated with Pam $3 \mathrm{CSK}_{4}+\mathrm{DA}-1229$, a beta cell-trophic effect of DPP4 inhibition was demonstrated by increased numbers of proliferating beta cells and small beta cell units. On the other hand, only a few apoptotic TUNEL ${ }^{+}$ beta cells were found in the pancreatic islets of prediabetic NOD mice, and this was unaffected by $\mathrm{Pam} 3 \mathrm{CSK}_{4}$ and/or DA-1229 (data not shown). While small beta cell units have been regarded as newly formed beta cells, representing beta cell neogenesis $[8,14]$, a recent paper argued against such a theory [37]. Progenitor cell recruitment and lineage tracing were also not assessed in this investigation. Hence, our results may not be sufficient to conclude that Pam $3 \mathrm{CSK}_{4}+\mathrm{DA}-1229$ increases beta cell neogenesis. 
While a suppressive effect of DPP4 inhibitors on serum glucagon level or glucagon secretion from alpha cells has been reported $[10,38]$, the effect of DPP4 inhibition on alpha cell mass has not been widely studied. Our results showing diminished alpha cell mass in diabetic NOD mice are consistent with a recent paper reporting reduced alpha cell mass in animal models of type 1 diabetes [39]. However, the decrement in alpha cell mass was lower than that in beta cells [39], which is consistent with the suppressed beta/alpha cell mass ratio in this study. The decrease in alpha cell mass in NOD mice could be caused by a paucity of local factors from beta cells necessary for alpha cell survival [39]. Our results are different from previous reports showing that DPP4 inhibitors attenuated the increase of alpha cell mass in diabetes induced by STZ and a high-fat diet [7, 38], and suggest that alpha cell changes associated with DPP4 inhibition vary depending on the experimental models or methods.

It was reported that DPP4 inhibitors, even without immune modulators, could reverse new-onset diabetes of NOD mice [36]. This effect has been attributed to the inhibition of CD26 (DPP4) and modulation of $\mathrm{T}$ cell migration $[33,36$, 40]. However, we did not observe significant effects of DPP4 inhibition in the model of established type 1 diabetes. Furthermore, DA-1229 alone did not prevent the development of diabetes in NOD mice (ESM Fig. 1), which is in contrast to the prevention of diabetes in NOD mice by $\mathrm{Pam}_{3} \mathrm{CSK}_{4}$ alone [4]. These results are consistent with the lack of DC tolerance in the PLNs of NOD mice treated with DA-1229 alone. The immunological mechanism underlying the different results is not clear, and could be ascribed to disparate effects of different DPP4 inhibitors. However, it is generally agreed that DPP4 inhibitors do not have significant immunological effects [41, 42]. To achieve significant inhibition of autoimmune process, other immunological measures will probably be necessary in addition to DPP4 inhibitors.

While not sufficient alone, immune modulation will be necessary for the treatment of clinically overt type 1 diabetes because remaining or new beta cells should be protected from autoimmune attack. TLR2 tolerance of DCs in NOD mice treated with $\mathrm{Pam} 3 \mathrm{CSK}_{4}+\mathrm{DA}-1229$ is essentially identical to that in NOD mice protected from the development of diabetes by Pam3CSK 4 [4]. While we showed that Treg cell change is not involved in the reversal of the model of established type 1 diabetes in NOD mice by $\mathrm{Pam}_{3} \mathrm{CSK}_{4}+\mathrm{DA}-1229$, a recent paper reported that two administrations of $\mathrm{Pam} 3 \mathrm{CSK}_{4}$ with a 5 day interval increased Treg cell number and ameliorated diabetes in NOD mice [43]. The different immunological impact of Pam $3 \mathrm{CSK}_{4}$ administration could be attributable to the different methods of administration.

Together, our results show that reversal of established diabetes is possible with a combination of TLR2 tolerisation and DPP4 inhibition, and suggest that DPP4 inhibitors could replace islet transplantation in this regard. The DPP4 inhibitor is one of the most popular drugs for the management of type 2 diabetes in the clinic. Pam $3 \mathrm{CSK}_{4}$ has also been used without specific side effects for the vaccination of cattle [44] and experimental animals [45, 46] and also in patients [47]. Thus, the method described here may provide a novel modality that could be applied in the clinic for the treatment of established type 1 diabetes without the issues associated with islet transplantation.

Acknowledgements DA-1229 was kindly provided by H.-J. Kim at Dong-A Pharmaceutical. We are grateful to E. Lee (Samsung Medical Center, Seoul, Korea) for assistance with English editing.

Funding This study was supported by the Bio Research \& Development Program (2008-04090) and the Korea Healthcare Technology Research \& Development Project, Ministry for Health, Welfare \& Family Affairs, Korea (A080967). M.-S. Lee is the recipient of a Global Research Laboratory Grant (K21004000003-10A0500-00310) and Bio \& Medical Technology Development Program (20110019335) of the National Research Foundation of Korea.

Duality of interest The authors declare that there is no duality of interest associated with this manuscript.

Contribution statement All authors contributed to the conception and design, or analysis and interpretation of data, drafting and revision of the article. D-HK and J-CL particularly contributed to the acquisition of experiment data. M-KL, K-WK and M-SL contributed to the design of the experiments, analysis of data and drafting of the manuscript, in particular. M-SL contributed to the initial conception of the study and to the supervision of all experiments throughout the study. All authors have approved the final version of the manuscript.

\section{References}

1. Trudeau JD, Dutz JP, Arany E, Hill DJ, Fieldus WE, Finegood DT (2000) Neonatal beta-cell apoptosis: a trigger for autoimmune diabetes? Diabetes 49:1-7

2. Turley S, Poirot L, Hattori M, Benoist C, Mathis D (2003) Physiological beta cell death triggers priming of self-reactive T cells by dendritic cells in a type-1 diabetes model. J Exp Med 198:1527-1537

3. Kim HS, Han MS, Chung KW et al (2007) Toll-like receptor 2 senses beta-cell death and contributes to the initiation of autoimmune diabetes. Immunity 27:321-333

4. Kim DH, Lee J-C, Kim S et al (2011) Inhibition of autoimmune diabetes by TLR2 tolerance. J Immunol 187:5211-5220

5. Baggio LL, Drucker DJ (1999) Biology of incretins: GLP-1 and GIP. Gastroenterology 132:2131-2157

6. Xu G, Stoffers DA, Habener JF, Bonner-Weir S (1999) Exendin-4 stimulates both beta-cell replication and neogenesis, resulting in increased beta-cell mass and improved glucose tolerance in diabetic rats. Diabetes 48:2270-2276

7. Mu J, Woods J, Zhou YP et al (2006) Chronic inhibition of dipeptidyl peptidase- 4 with a sitagliptin analog preserves pancreatic beta-cell mass and function in a rodent model of type 2 diabetes. Diabetes 55:1695-1704

8. Pospisilik JA, Martin J, Doty T et al (2003) Dipeptidyl peptidase IV inhibitor treatment stimulates beta-cell survival and islet neogenesis in streptozotocin-induced diabetic rats. Diabetes 52:741-750 
9. Mest HJ, Mentlein R (2005) Dipeptidyl peptidase inhibitors as new drugs for the treatment of type 2 diabetes. Diabetologia 48:616-620

10. Cho JM, Jang HW, Cheon H et al (2011) A novel dipeptidyl peptidase IV inhibitor DA-1229 ameliorates streptozotocininduced diabetes by increasing $\beta$-cell replication and neogenesis. Diabetes Res Clin Pract 91:72-79

11. Suk K, Kim S, Kim Y-H et al (2001) IFN $\gamma / \mathrm{TNF} \alpha$ synergism as the final effector in autoimmune diabetes: a key role for STAT1/IRF-1 in pancreatic $\beta$-cell death. J Immunol 166:4481-4489

12. Kim Y-H, Kim S, Kim K-A et al (1999) Apoptosis of pancreatic $\beta$-cells detected in accelerated diabetes of NOD mice: no role of Fas-Fas ligand interaction in autoimmune diabetes. Eur J Immunol 29:455-465

13. Jung HS, Chung KW, Kim JW et al (2008) Loss of autophagy diminishes pancreatic $\beta$-cell mass and function with resultant hyperglycemia. Cell Metab 8:318-324

14. Bouwens L, Pipeleers DG (1998) Extra-insular beta cells associated with ductules are frequent in adult human pancreas. Diabetologia 41:629-633

15. Han MS, Chung KW, Cheon HG et al (2009) Imatinib mesylate reduces endoplasmic reticulum stress and induces remission of diabetes in $\mathrm{db} / \mathrm{db}$ mice. Diabetes 58:329-336

16. Tang Q, Adams JY, Penaranda C et al (2008) Central role of defective interleukin-2 production in the triggering of islet autoimmune destruction. Immunity 28:687-697

17. Chatenoud L, Bluestone JA (2007) CD3-specific antibodies: a portal of the treatment of autoimmunity. Nat Immunol 7:622-632

18. Orban T, Bundy B, Becker DJ et al (2011) Co-stimulation modulation with abatacept in patients with recent-onset type 1 diabetes: a randomised, double-blind, placebo-controlled trial. Lancet 378:412-419

19. $\mathrm{Hu} \mathrm{C}$, Rodriguez-Pinto D, Du W et al (2007) Treatment with CD20 -specific antibody prevents and reverse autoimmune diabetes in mice. J Clin Invest 117:3857-3867

20. Morris MA, McDuffie M, Nadler JL, Ley K (2011) Prevention, but not cure, of autoimmune diabetes in a NOD.scid transfer model by FTY720 despite effective modulation of blood T cells. Autoimmunity 44:115-128

21. Mastrandrea L, Yu J, Behrens T et al (2009) Etanercept treatment in children with new-onset type 1 diabetes. Diabetes Care 32:1244-1249

22. Tarbell KV, Petit L, Zuo X et al (2007) Dendritic cell-expanded, islet-specific $\mathrm{CD} 4+\mathrm{CD} 25+\mathrm{CD} 62 \mathrm{~L}+$ regulatory $\mathrm{T}$ cells restore normoglycemia in diabetic NOD mice. J Exp Med 204:191-201

23. Hasse C, Yu L, Eisenbarth G, Harkholst H (2010) Antigendependent immunotherapy of non-obese diabetic mice with immature dendritic cells. Clin Exp Immunol 160:331-339

24. Nussbaum G, Zanin-Zhorov A, Quintana F, Lider O, Cohen IR (2006) Peptide p277 of HSP60 signals T cells: inhibition of inflammatory chemotaxis. Int Immunol 18:1413-1419

25. Allen HF, Klingensmith GJ, Jensen PE, Simoes E, Hayward A, Chase HP (1999) Effect of Bacillus Calmette-Guerin vaccination on new-onset type 1 diabetes. Diabetes Care 22:1703-1707

26. Group TDS (2002) Effects of insulin in relatives of patients with type 1 diabetes mellitus. N Engl J Med 202:346

27. Ludvigsson J, Faresjö M, Hjorth M et al (2008) GAD treatment and insulin secretion in recent-onset type 1 diabetes. N Engl J Med 359:1909-1920

28. Alipio Z, Liao W, Roemer EJ et al (2010) Reversal of hyperglycemia in diabetic mouse models using induced-pluripotent stem (iPS)-derived pancreatic beta-like cells. Proc Natl Acad Sci USA 107:13426-13431

29. Agudo J, Ayuso E, Jimenez V et al (2008) IGF-I mediates regeneration of endocrine pancreas by increasing beta cell replication through cell cycle protein modulation in mice. Diabetologia 52:1862-1872

30. Lavine JA, Raess PW, Davis DB et al (2008) Overexpression of pre-pro-cholecystokinin stimulates beta-cell proliferation in mouse and human islets with retention of islet function. Mol Endocrinol $22: 2716-2728$
31. Ogawa N, List JF, Habener JF, Maki T (2004) Cure of overt diabetes in NOD mice by transient treatment with antilymphocyes serum and exentin-4. Diabetes 53:1700-1705

32. Sherry NA, Chen W, Kushner JA et al (2007) Exendin-4 improves reversal of diabetes in NOD mice treated with anti-CD-3 monoclonal antibody by enhancing recovery of $\beta$-cells. Endocrinology 148:5136-5144

33. Suarez-Pinzon WL, Cembrowski GS, Rabinovitch A (2009) Combination therapy with a dipeptidyl peptidase-4 inhibitor and a proton pump inhibitor restores normoglycemia in non-obese diabetic mice. Diabetologia 52:1680-1682

34. Suarez-Pinzon WL, Lakey JR, Brand SJ, Rabinovitch A (2005) Combination therapy with epidermal growth factor and gastrin induces neogenesis of human islet $\beta$-cells from pancreatic duct cells and an increase in functional $\beta$-cell mass. J Clin Endocrinol Metab 90:3401-34091

35. Suarez-Pinzon WL, Power RF, Yan Y, Wasserfall C, Atkinson M, Rabinovitch A (2008) Combination therapy with glucagon-like peptide-1 and gastrin restores normoglycemia in diabetic NOD mice. Diabetes 57:3281-3288

36. Tian L, Gao J, Hao J et al (2010) Reversal of new-onset diabetes through modulating inflammation and stimulating $\beta$-cell replication in nonobese diabetic mice by a dipeptidyl peptidase IV inhibitor. Endocrinology 151:3049-3060

37. Dor Y, Brown J, Martinez OI, Melton DA (2004) Adult pancreatic beta-cells are formed by self-duplication rather than stem-cell differentiation. Nature 429:41-46

38. Dunning BE, Foley JE, Ahren B (2005) Alpha cell function in health and disease: influence of glucagon-like peptide-1. Diabetologia 48:1700-1713

39. Pechhold K, Zhu X, Harrison VS et al (2009) Dynamic changes in pancreatic endocrine cell abundance, distribution, and function in antigen-induced and spontaneous autoimmune diabetes. Diabetes 58:1175-1184

40. Kim S-J, Nian C, Doudet DJ, McIntosh CH (2009) Dipeptidyl peptidase IV inhibition with MK0431 improves islet graft survival in diabetic NOD mice partially via $\mathrm{T}$ cell modulation. Diabetes 58:641-651

41. Reinhold D, Goihl A, Wrenger S et al (2009) Role of dipeptidyl peptidase IV (DPPIV)-like enzymes in T lymphocyte activation: investigations in DPPIV/CD26-knockout mice. Clin Chem Lab Med 47:268-274

42. Vora KA, Porter G, Peng R et al (2009) Genetic ablation or pharmacological blockade of dipeptidyl peptidase IV does not impact $\mathrm{T}$ celldependent immune responses. BMC Immunol 10:1-11

43. Filippi CM, Ehrhartd K, Estes EA, Larsson P, Oldham JE, von Herrath M (2011) TLR2 signaling improves immunoregulation to prevent type 1 diabetes. Eur J Immunol 41:1399-1409

44. Höhlich BJ, Wiesmüller KH, Haas B et al (2003) Induction of an antigen-specific immune response and partial protection of cattle against challenge infection with foot-and-mouth disease virus (FMDV) after lipopeptide vaccination with FMDV-specific B cell epitopes. J Gen Virol 84:3315-3324

45. Kudryashov V, Glunz PW, Williams LJ, Hintermann S, Danishefsky D, Lloyd KO (2001) Toward optimized carbohydrate-based anticancer vaccines: epitope clustering, carrier structure, and adjuvant all influence antibody responses to Lewisy conjugates in mice. Proc Natl Acad Sci USA 98:3264-3269

46. Zhu X, Ramos TV, Gras-Masse H, Kaplan BE, BenMohamed L (2004) Lipopeptide epitopes extended by an N-palmitoyl-lysine moiety increase uptake and maturation of dendritic cells through a Toll-like receptor-2 pathway and trigger a Th1-dependent protective immunity. Eur J Immunol 34:3102-3114

47. Seth A, Yasutomi Y, Jacoby H et al (2000) Evaluation of a lipopeptide immunogen as a therapeutic in HIV type 1-seropostivie individuals. AIDS Res Hum Retroviruses 16:337-343 